\title{
SMALL-NOISE LIMIT OF THE QUASI-GAUSSIAN LOG-NORMAL HJM MODEL
}

\author{
DAN PIRJOL AND LINGJIONG ZHU
}

\begin{abstract}
Quasi-Gaussian HJM models are a popular approach for modeling the dynamics of the yield curve. This is due to their low dimensional Markovian representation, which greatly simplifies their numerical implementation. We present a qualitative study of the solutions of the quasi-Gaussian log-normal HJM model. Using a small-noise deterministic limit we show that the short rate may explode to infinity in finite time. This implies the explosion of the Eurodollar futures prices in this model. We derive explicit explosion criteria under mild assumptions on the shape of the yield curve.
\end{abstract}

\section{INTRODUCTION}

HJM models [10] are widely used in financial practice for modeling fixed income, credit and commodity markets [1]. These models specify the dynamics of the yield curve $f(t, T)$ as

$$
d f(t, T)=\sigma_{f}(t, T)^{T} d W(t)+\sigma_{f}^{T}(t, T)\left(\int_{t}^{T} \sigma_{f}(t, s) d s\right) d t,
$$

where $W(t)$ is a vector Brownian motion under the risk-neutral measure $\mathbb{Q}$ and $\left\{\sigma_{f}(t, T)\right\}_{t \leq T}$ is a family of vector processes. The numerical simulation of these models is complicated by the fact that the entire yield curve $f(t, T)$ has to be simulated. Lattice and tree simulation methods require an exponentially large number of nodes. For this reason the simulation of these models is restricted in practice to Monte Carlo methods.

The quasi-Gaussian HJM models $[1,2,3,5,15]$ were introduced to simplify the simulation of the HJM models. They are obtained by assuming a separable form for the volatility $\sigma_{f}(t, T)^{T}=$ $g(T)^{T} h(t)$ where $g$ is a deterministic vector function and $h$ is a $k \times k$ matrix process. Such models admit a Markov representation of the dynamics of the yield curve involving $k+\frac{1}{2} k(k+1)$ state variables. This simplifies very much their simulation, which can be done either using Monte Carlo or finite difference methods $[4,8]$.

We consider in this note the one-factor quasi-Gaussian HJM model with volatility specification $\sigma_{f}(t, T)=k(t, T) \sigma\left(r_{t}\right)$ where $k(t, T)=e^{-\beta(T-t)}$, and $\sigma\left(r_{t}\right)$ is the volatility of the short rate $r_{t}=$ $f(t, t)$. This model admits a two state Markov representation.

It has been noted in $[13,10]$ that in HJM models with log-normal volatility specification, that is for which $\sigma_{f}(t, T)=\sigma(t, T) f(t, T)$, the rates explode to infinity with probability one, and zero coupon bond prices are zero. It is natural to ask if a similar explosive phenomenon is present also in the quasi-Gaussian HJM model with log-normal volatility $\sigma\left(r_{t}\right)=\sigma r_{t}$. This model is used

Date: 20 October 2016.

Key words and phrases. HJM model, explosion, stochastic modeling, ordinary differential equations. 
in financial practice for modeling swaption volatility smiles [6] and is a particular case of a more general parametric representation [7].

We study in this note the qualitative behavior of the solutions of this model. In the small-noise deterministic limit, we show rigorously that the short rate may explode to infinity in a finite time. More precisely, for sufficiently small mean-reversion $\beta$, the deterministic approximation for the short rate has an explosion in finite time, and an upper bound is given on the explosion time, which is saturated in the flat forward rate limit. When Brownian noise is taken into account, the explosion time has a distribution around the deterministic limit.

This phenomenon has implications for the practical use of the model for pricing and simulation. It implies an explosion of the Eurodollar futures prices in this model, and introduces a limitation in the applicability of the model for pricing these products to maturities smaller than the explosion time.

\section{Log-NORMAL QUASI-Gaussian HJM MODEL}

The one-factor log-normal quasi-Gaussian HJM model is defined by the volatility specification

$$
\sigma_{f}(t, T)=\sigma r_{t} e^{-\beta(T-t)} .
$$

The simulation of the model requires the solution of the stochastic differential equation for the two variables $\left\{x_{t}, y_{t}\right\}_{t \geq 0}[15,1]$

$$
\begin{aligned}
& d x_{t}=\left(y_{t}-\beta x_{t}\right) d t+\sigma\left(\lambda(t)+x_{t}\right) d W_{t}, \\
& d y_{t}=\left(\sigma^{2}\left(\lambda(t)+x_{t}\right)^{2}-2 \beta y_{t}\right) d t,
\end{aligned}
$$

with initial condition $x_{0}=y_{0}=0$. Here $\lambda(t)=f(0, t)$ is the forward short rate, giving the initial yield curve. The zero coupon bonds are

$$
P(t, T)=\frac{P(0, T)}{P(0, t)} \exp \left(-G(t, T) x_{t}-\frac{1}{2} G^{2}(t, T) y_{t}\right),
$$

with $G(t, T) \geq 0$ a non-negative deterministic function [1]. The short rate is $r_{t}:=f(t, t)=\lambda(t)+x_{t}$. The equations (3) can be expressed in terms of the short rate as

$$
\begin{aligned}
& d r_{t}=\left(y_{t}-\beta r_{t}+\beta \lambda(t)+\lambda^{\prime}(t)\right) d t+\sigma r_{t} d W_{t}, \\
& d y_{t}=\left(\sigma^{2} r_{t}^{2}-2 \beta y_{t}\right) d t
\end{aligned}
$$

with the initial condition $r_{0}=\lambda_{0}:=\lambda(0)>0$ and $y_{0}=0$.

The solutions of the process (5) may explode with non-zero probability. This will be discussed in a future paper [14]. When the volatility $\sigma=0$, there is no explosion. Indeed, when $\sigma=0$, we have

$$
\begin{aligned}
d r_{t} & =\left(y_{t}-\beta r_{t}+\beta \lambda(t)+\lambda^{\prime}(t)\right) d t, \\
d y_{t} & =-2 \beta y_{t} d t,
\end{aligned}
$$

with the initial condition $r_{0}=\lambda_{0}$ and $y_{0}=0$. Thus $y_{t} \equiv 0$, which gives $r_{t}^{\prime}=-\beta r_{t}+\beta \lambda(t)+\lambda^{\prime}(t)$. This ODE can be easily solved with the result $r_{t}=\lambda(t)$. 


\section{Deterministic Approximation}

Instead of studying directly the distribution of the explosion time of the process $\left(r_{t}, y_{t}\right)$, we study a deterministic proxy of the equations (5). In the limit when the Brownian noise in these equations goes to zero, then $\left(r_{t}, y_{t}\right) \rightarrow(r(t), y(t))$, where $(r(t), y(t))$ satisfy the two-dimensional ODE:

$$
\begin{aligned}
& r^{\prime}(t)=y(t)-\beta r(t)+\beta \lambda(t)+\lambda^{\prime}(t), \\
& y^{\prime}(t)=\sigma^{2} r^{2}(t)-2 \beta y(t),
\end{aligned}
$$

with $r(0)=\lambda_{0}$ and $y(0)=0$. The variable $r(t)$ can be interpreted as the deterministic approximation of the short rate $r_{t}$ and its expected value $\mathbb{E}^{\mathbb{Q}}\left[r_{t}\right]$ for the small-noise limit. The pair $(r(t), y(t))$ is a deterministic approximation of the two-dimensional SDE (5).

We study here the qualitative properties of the solution for $r(t)$. Even though (6) is a system of 2D ODEs, we will show that $r(t)$ can be expressed as a solution to a $1 \mathrm{D}$ integral equation.

Proposition 1. $r(t)$ satisfies the integral equation

$$
r(t)=\lambda(t)+\frac{\sigma^{2}}{\beta} \int_{0}^{t} r^{2}(s)\left[e^{\beta(s-t)}-e^{2 \beta(s-t)}\right] d s .
$$

Proof. We can solve for $y(t)$ as

$$
y(t)=\sigma^{2} \int_{0}^{t} r^{2}(s) e^{2 \beta(s-t)} d s
$$

Substituting into (6) we get

$$
r^{\prime}(t)+\beta r(t)=\sigma^{2} \int_{0}^{t} r^{2}(s) e^{2 \beta(s-t)} d s+\beta \lambda(t)+\lambda^{\prime}(t) .
$$

Multiplying by the integrating factor $e^{\beta t}$ and integrating from 0 to $t$, we obtain:

$$
\begin{aligned}
r(t) e^{\beta t}-\lambda_{0} & =\sigma^{2} \int_{0}^{t} \int_{0}^{u} r^{2}(s) e^{2 \beta s} e^{-\beta u} d u d s+\lambda(t) e^{\beta t}-\lambda_{0} \\
& =\lambda(t)+\sigma^{2} \int_{0}^{t} \int_{s}^{t} r^{2}(s) e^{2 \beta s} e^{-\beta u} d u d s-\lambda_{0},
\end{aligned}
$$

which yields Eq. (7).

We show next that if $\lambda(t)$ is uniformly bounded, for sufficiently large $\beta$ or sufficiently small $\sigma, r(t)$ is also uniformly bounded, and hence there will be no explosion.

Proposition 2. Assume that $\lambda(t)$ is uniformly bounded. Then, for sufficiently large $\beta$ or sufficiently small $\sigma$, we have

$$
\max _{t \geq 0} r(t) \leq \frac{\beta^{2}}{\sigma^{2}}\left(1-\sqrt{1-\max _{t \geq 0} \lambda(t) \frac{2 \sigma^{2}}{\beta^{2}}}\right) .
$$

It follows that there will be no explosion.

Proof. We only give a proof for the large $\beta$ result. The same result holds for sufficiently small $\sigma$ with a very similar proof. 
For any $t \in[0, T]$, we have from Eq. (7)

$$
r(t) \leq \max _{0 \leq t \leq T} \lambda(t)+\left[\max _{0 \leq t \leq T} r(t)\right]^{2} \frac{\sigma^{2}}{\beta} \int_{0}^{\infty}\left[e^{-\beta s}-e^{-2 \beta s}\right] d s,
$$

which implies that $R(T):=\max _{0 \leq t \leq T} r(t)$ satisfies

$$
R(T)-R^{2}(T) \frac{\sigma^{2}}{2 \beta^{2}} \leq \max _{0 \leq t \leq T} \lambda(t) .
$$

This implies that we have either (i) $R(T) \leq R_{1}(T)$, or (ii) $R(T) \geq R_{2}(T)$, with

$$
R_{1,2}(T):=\frac{\beta^{2}}{\sigma^{2}}\left(1 \mp \sqrt{1-\max _{0 \leq t \leq T} \lambda(t) \frac{2 \sigma^{2}}{\beta^{2}}}\right) .
$$

For large $\beta, R(T)$ is bounded by Proposition 2, while $R_{2}(T) \rightarrow \infty$ as $\beta \rightarrow \infty$. Therefore, for sufficiently large $\beta>0$, we have $R(T) \leq R_{1}(T)$. Taking now $T \rightarrow \infty$, we have by the uniformly bounded assumption $\max _{t \geq 0} \lambda(t)<\infty$. It follows that for sufficiently large $\beta$,

$$
\max _{t \geq 0} r(t) \leq \frac{\beta^{2}}{\sigma^{2}}\left(1-\sqrt{1-\max _{t \geq 0} \lambda(t) \frac{2 \sigma^{2}}{\beta^{2}}}\right) .
$$

We conclude that for sufficiently large $\beta, r(t)$ is not explosive and is indeed uniformly bounded as long as $\lambda(t)$ is uniformly bounded.

Remark 1. From Proposition 2, it follows that $\max _{t \geq 0} r(t)$ is uniformly bounded as either $\beta \rightarrow \infty$ or $\sigma \rightarrow 0$, since

$$
\limsup _{\beta \rightarrow \infty} \max _{t \geq 0} r(t) \leq \limsup _{\beta \rightarrow \infty} \frac{\beta^{2}}{\sigma^{2}}\left(1-\sqrt{1-\max _{t \geq 0} \lambda(t) \frac{2 \sigma^{2}}{\beta^{2}}}\right)=\max _{t \geq 0} \lambda(t),
$$

and the same result holds for $\sigma \rightarrow 0$.

From Proposition 1, Proposition 2 and Remark 1, we immediately get the following corollary.

Corollary 1. As $\beta \rightarrow \infty$ (resp. $\sigma \rightarrow 0$ ), we have $r(t) \rightarrow \lambda(t)$ uniformly for $t \geq 0$. More precisely,

$$
\left.\max _{t \geq 0}|r(t)-\lambda(t)| \leq\left[\max _{t \geq 0} \lambda(t)\right]^{2} \frac{\sigma^{2}}{2 \beta^{2}}+o\left(\beta^{-2}\right) \quad\left(\text { resp. o }\left(\sigma^{2}\right)\right), \quad \text { as } \beta \rightarrow \infty \text { (resp. } \sigma \rightarrow 0\right) .
$$

Proof. From Proposition 1, Proposition 2 and Remark 1, we get

$$
\begin{aligned}
\max _{t \geq 0}|r(t)-\lambda(t)| & \leq \max _{t \geq 0} \frac{\sigma^{2}}{\beta} \int_{0}^{t} r^{2}(t-s)\left[e^{-\beta s}-e^{-2 \beta s}\right] d s \\
& \leq\left[\max _{t \geq 0} r(t)\right]^{2} \frac{\sigma^{2}}{\beta} \int_{0}^{\infty}\left[e^{-\beta s}-e^{-2 \beta s}\right] d s \\
& \leq\left[\max _{t \geq 0} \lambda(t)\right]^{2} \frac{\sigma^{2}}{2 \beta^{2}}+o\left(\beta^{-2}\right) \quad\left(\text { resp. } o\left(\sigma^{2}\right)\right), \quad \text { as } \beta \rightarrow \infty(\text { resp. } \sigma \rightarrow 0) .
\end{aligned}
$$

We can also study the stationary limit for the deterministic system, that is, the large time limit for the deterministic system. 
Proposition 3. Let us assume that $\lim _{t \rightarrow \infty} \lambda(t)=\lambda(\infty)$ and $\lim _{t \rightarrow \infty} r(t)=r(\infty)$ exist, then

$$
r(\infty)=\frac{\beta^{2}}{\sigma^{2}}\left(1-\sqrt{1-\lambda(\infty) \frac{2 \sigma^{2}}{\beta^{2}}}\right) .
$$

Proof. Let us recall that

$$
r(t)=\lambda(t)+\frac{\sigma^{2}}{\beta} \int_{0}^{t}(r(t-s))^{2}\left[e^{-\beta s}-e^{-2 \beta s}\right] d s .
$$

Thus the large time limit $r(\infty)$ is the smaller root of the equation

$$
r(\infty)=\lambda(\infty)+r(\infty)^{2} \frac{\sigma^{2}}{\beta} \int_{0}^{\infty}\left[e^{-\beta s}-e^{-2 \beta s}\right] d s,
$$

which yields (16).

For constant $\lambda(t) \equiv \lambda_{0}$, one can study the stability of the two-dimensional first-order ODE

$$
\begin{aligned}
r^{\prime}(t) & =y(t)-\beta r(t)+\beta \lambda_{0}, \\
y^{\prime}(t) & =\sigma^{2} r^{2}(t)-2 \beta y(t) .
\end{aligned}
$$

We would like to determine the fixed points of this equation, and determine their type. The fixed points are given by the zeros of the functions on the RHS of the ODE. For $\beta^{2}<\beta_{C}^{2}:=2 \lambda_{0} \sigma^{2}$ there are no fixed points. For $\beta^{2}=\beta_{C}^{2}$ there is one at $r_{1}=2 \lambda_{0}, y_{1}=\lambda_{0} \beta_{C}$. For $\beta^{2}>\beta_{C}^{2}$ there are two fixed points:

$$
\Pi_{1,2}: r_{1,2}=\frac{\beta^{2}}{\sigma^{2}}(1 \mp \sqrt{\Delta}), \quad y_{1,2}=\frac{\beta^{3}}{2 \sigma^{2}}(1 \mp \sqrt{\Delta})^{2} .
$$

with $\Delta:=1-\frac{2 \lambda_{0} \sigma^{2}}{\beta^{2}}$. Linearization of the ODE around each fixed point gives the linear ODE

$$
\left(\begin{array}{c}
r^{\prime}(t) \\
y^{\prime}(t)
\end{array}\right)=\left(\begin{array}{cc}
-\beta & 1 \\
2 \sigma^{2} r_{i} & -2 \beta
\end{array}\right) \cdot\left(\begin{array}{c}
r(t)-r_{i} \\
y(t)-y_{i}
\end{array}\right)+\text { higher order terms }
$$

The eigenvalues of the matrix of coefficients for each fixed point are:

$$
\begin{aligned}
& \Pi_{1} \quad: \quad \lambda_{1,2}=\frac{1}{2}\left(-3 \beta \pm 3 \beta \sqrt{1-\frac{8}{9} \sqrt{\Delta}}\right), \\
& \Pi_{2} \quad: \quad \lambda_{1,2}=\frac{1}{2}\left(-3 \beta \pm 3 \beta \sqrt{1+\frac{8}{9} \sqrt{\Delta}}\right) .
\end{aligned}
$$

The relative signs of the two eigenvalues determine the type of the fixed points: $\Pi_{1}$ is an attractive fixed point $\left(\lambda_{1}, \lambda_{2}<0\right)$, and $\Pi_{2}$ is a saddle point $\left(\lambda_{1}>0, \lambda_{2}<0\right)$. This means that the twodimensional ODE describes a flow for $(r(t), y(t))$, and the lines of flow can either end at $\Pi_{1}$, or at infinity, avoiding $\Pi_{2}$. 


\section{EXPLOSION CRITERIA}

We study in this Section in more detail the explosion time of $r(t)$, defined as $\tau_{\infty}:=\sup \{\tau: r(\tau)<$ $\infty\}$. The starting point of the analysis is the 2nd order ODE for $r(t)$

$$
r^{\prime \prime}(t)+3 \beta r^{\prime}(t)+2 \beta^{2} r(t)=\sigma^{2} r^{2}(t)+2 \beta^{2} \lambda_{0}+\Lambda(t)
$$

where we defined $\Lambda(t):=2 \beta^{2}(\lambda(t)-\lambda(0))+3 \beta \lambda^{\prime}(t)+\lambda^{\prime \prime}(t)$.

We will make the following assumption about the initial forward rate $\lambda(t)$.

Assumption 1. Assume that $\lambda(t)$ satisfies the condition

$$
\Lambda(t):=2 \beta^{2}(\lambda(t)-\lambda(0))+3 \beta \lambda^{\prime}(t)+\lambda^{\prime \prime}(t) \geq 0, \quad t \geq 0 .
$$

This is satisfied by forward rate curves $\lambda(t)$ which are flat or up-sloping and not too concave, which are usual in normal market conditions.

Under this assumption, $r(t)$ satisfies the differential inequality, which reduces to equality in the limit of a constant $\lambda(t)=\lambda_{0}$

$$
r^{\prime \prime}(t)+3 \beta r^{\prime}(t)+2 \beta^{2} r(t) \geq \sigma^{2} r^{2}(t)+2 \beta^{2} \lambda_{0},
$$

with initial conditions

$$
r(0)=\lambda_{0}, \quad r^{\prime}(0)=0
$$

4.1. Zero mean-reversion case $\beta=0$.

Proposition 4. The explosion time of $r(t)$ in the one-factor log-normal quasi-Gaussian HJM model under the Assumption 1 with $\beta=0$ is bounded from above as

$$
\tau_{\infty} \leq \frac{\sqrt{6 p_{0}} \omega_{2}}{\sigma \sqrt{\lambda_{0}}}=\frac{2.97448}{\sigma \sqrt{\lambda_{0}}}
$$

where $p_{0}=0.62996, \omega_{2}=1.52995$ are parameters of the Weierstrass elliptic function [16]. The inequality in (29) becomes sharp when $\lambda(t) \equiv \lambda_{0}$.

Proof. Taking $\beta=0$ in (25) we get the differential inequality

$$
r^{\prime \prime}(t) \geq \sigma^{2} r^{2}(t)
$$

with initial conditions (28).

By Lemma 1 (see Appendix) it is sufficient to study the solution of the ODE obtained by replacing the inequality sign in (30) with equality, and satisfying the same initial condition at $t=0$. The solution of this equation $Z^{\prime \prime}(t)=\sigma^{2} Z^{2}(t)$ with $Z(0)=\lambda_{0}, Z^{\prime}(0)=0$ can be found exactly in terms of the Weierstrass elliptic function $\wp\left(z ; c_{1}, c_{2}\right)$, and is given by

$$
Z(t)=\frac{6^{1 / 3}}{\sigma^{2 / 3}} \wp\left(\left(\frac{\sigma^{2}}{6}\right)^{1 / 3}\left(t+c_{1}\right) ; 0, c_{2}\right),
$$

where the constants $c_{1}, c_{2}$ are given by

$$
c_{1}=\sqrt{6 p_{0}} \omega_{2} \frac{1}{\sigma \sqrt{\lambda_{0}}}, \quad c_{2}=\frac{1}{6 p_{0}^{3}} \lambda_{0}^{3} \sigma^{2},
$$

with $p_{0}=0.62996, \omega_{2}=1.52995$. This can be simplified further by using the relation [16] $\wp\left(z ; 0, c_{2}\right)=c_{2}^{1 / 3} \wp\left(z c_{2}^{1 / 6} ; 0,1\right)$ to rescale the second argument $c_{2}$ to 1 . 


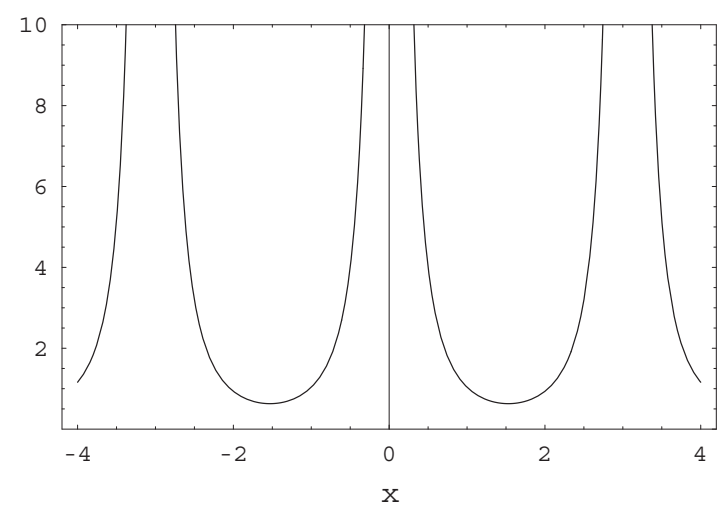

Figure 1. Plot of the Weierstrass elliptic function $\wp(x ; 0,1)$.

The main features of the solution can be understood by recalling the main properties of $\wp(z ; 0,1)$ [16]. Along the real axis this function is periodic with half-period $\omega_{2} \simeq 1.52995$, and has double poles at origin $z=0$ and at all points uniformly spaced by $2 \omega_{2}$. Half-way between poles at $z=\omega_{2}$ it reaches a minimum value $p_{0}=0.62996$. The plot of $\wp(z ; 0,1)$ is shown in Fig. 1.

Time zero corresponds to $z=\omega_{2}$ and the explosion of $r(t)$ takes place at the nearest pole, at $z=2 \omega_{2}$. This gives for the explosion time of the solution $Z(t)$, defined as $\bar{\tau}_{\infty}:=\sup \{t: Z(t)<\infty\}$

$$
\bar{\tau}_{\infty}=c_{1}=\frac{1}{\sigma \sqrt{\lambda_{0}}} \sqrt{6 p_{0}} \omega_{2}=2.97448 \frac{1}{\sigma \sqrt{\lambda_{0}}} .
$$

For constant forward rate $\lambda(t)=\lambda_{0}$ we have $\tau_{\infty}=\bar{\tau}_{\infty}$. Allowing for non-constant $\lambda(t)$ satisfying Assumption 1 we have the upper bound on the explosion time $\tau_{\infty} \leq \bar{\tau}_{\infty}$.

As a numerical illustration we consider the following typical model parameters: constant forward rate $\lambda_{0}=5 \%$ and volatility $\sigma=20 \%$. The explosion time of the short rate $r(t)$ is $\tau_{\infty}=66.5 Y$. This decreases as the volatility $\sigma$ increases or the forward rate $\lambda_{0}$ increases.

4.2. Positive mean-reversion case $\beta>0$. It was shown in Proposition 2 that in the limit $\beta \rightarrow \infty$ there is no explosion in $r(t)$. On the other hand, for $\beta=0$ there is explosion (Proposition 4). This implies that as $\beta$ increases from zero, there is a maximum value of $\beta$ for which there is explosion. For constant $\lambda(t)=\lambda_{0}$ this maximum value can be found explicitly, and is given by the following result. For $\beta$ less than this maximum value, the result can be extended to a wider class of functions $\lambda(t)$, satisfying Assumption 1.

Proposition 5. The critical value $\beta_{C}:=\sigma \sqrt{2 \lambda_{0}}$ separates the solutions of (25) into two families, with different qualitative behavior:

(i) Small mean-reversion $\beta<\beta_{\mathrm{C}}$. Under the Assumption 1 the explosion time of the solution for $r(t)$ is bounded from above as $\tau_{\infty} \leq \bar{\tau}_{\infty}$ with

$$
\bar{\tau}_{\infty}=\int_{\lambda_{0}}^{\infty} \frac{d x}{\sqrt{y(x)}}
$$

where $y(x)$ is the solution of the first order nonlinear $O D E$

$$
\frac{1}{2} y^{\prime}(x)+3 \beta \sqrt{y(x)}=\sigma^{2} x^{2}-2 \beta^{2} x+2 \beta^{2} \lambda_{0},
$$


with initial condition $y\left(\lambda_{0}\right)=0$. The inequality $\tau_{\infty} \leq \bar{\tau}_{\infty}$ becomes sharp for $\lambda(t)=\lambda_{0}$.

(ii) Large mean-reversion $\beta \geq \beta_{\mathrm{C}}$. Assuming constant $\lambda(t)=\lambda_{0}$, the solution for $r(t)$ does not reach infinity. It satisfies $\lim _{t \rightarrow \infty} r(t)=x_{1}$ with $x_{1}$ given by (38). For general $\lambda(t)$ a numerical study is required in order to decide the absence or presence of an explosion for $r(t)$.

Proof. We start by assuming $\lambda(t)=\lambda_{0}$, and prove the equation (35). For sufficiently small $\beta, r(t)$ is a strictly increasing function of $t$ and can be inverted. Denote $v(x):=r^{\prime}\left(r^{-1}(x)\right)$. It is easy to see that this satisfies

$$
v^{\prime}(x)=\frac{d}{d x} v(x)=r^{\prime \prime}(t) \frac{1}{r^{\prime}(t)} .
$$

Multiplying with $v(x)$ and using (25) we get that this function satisfies the differential equation

$$
v(x) v^{\prime}(x)+3 \beta v(x)=\sigma^{2} x^{2}-2 \beta^{2} x+2 \beta^{2} \lambda_{0} .
$$

(With the substitution $z=-3 \beta x$, the equation (37) can be brought into the canonical form of the Abel equation of the second kind $v(z) v^{\prime}(z)-v(z)=f(z)$.) This equation is difficult to simulate numerically as its behavior near $x=\lambda_{0}$ is singular, i.e. $\lim _{x \rightarrow \lambda_{0}} v^{\prime}(x)=\infty$. It is more convenient to define $y(x)=v^{2}(x)$, which satisfies (35) and has well-behaved behavior $\lim _{x \rightarrow \lambda_{0}} y^{\prime}(x)=2 \sigma^{2} \lambda_{0}^{2}$. This reproduces Eq. (35).

We would like to study the finiteness of the integral in (34). For sufficiently small positive $\beta$, the function $v(x)=\sqrt{y(x)}$ is everywhere positive. Its large $x$ asymptotics is $v(x)=c_{1} x^{3 / 2}+O\left(x^{1 / 2}\right)$, so the integral (34) converges at the upper limit of integration. However, we will show that as $\beta$ increases, the solution $y(x)$ develops a zero at a point $x_{0}$ as $\beta$ approaches a certain value $\beta_{\mathrm{C}}$. At this point the integral (34) diverges.

Let us consider the behavior of the solution $v(x)$ as $\beta$ is increased from zero. Denote $f(x):=$ $\sigma^{2} x^{2}-2 \beta^{2} x+2 \beta^{2} \lambda_{0}$ the function on the right hand side of (35) (with $\left.\Lambda(t)=0\right)$ ). $f(x)$ is always positive for $\beta<\beta_{C}=\sigma \sqrt{2 \lambda_{0}}$ and becomes negative in a region $\left(x_{1}, x_{2}\right)$ for $\beta>\beta_{C}$, with

$$
x_{1,2}=\frac{\beta^{2}}{\sigma^{2}}\left(1 \pm \sqrt{1-\frac{2 \sigma^{2} \lambda_{0}}{\beta^{2}}}\right) .
$$

For $\beta=\beta_{C}$ the function $f(x)$ vanishes at $x_{1}=x_{2}=2 \lambda_{0}$. These solutions satisfy the inequalities $x_{1,2}>\lambda_{0}$. As $\beta \rightarrow \infty$, the smallest solution approaches $\lambda_{0}$ from above: $\lim _{\beta \rightarrow \infty} x_{1}=\lambda_{0}$. The points $x_{1,2}$ are fixed points for the equation (25), and correspond to the fixed points $\Pi_{1,2}$ for the system $(r(t), y(t))$ studied in Section 3. Recall that $x_{1}$ is a stable fixed point, and $x_{2}$ is a saddle point.

We note the following properties of the solution $y(x)$ of the equation (35):

(1) $y(x)$ is an increasing function in a neighborhood of $x=\lambda_{0}$. This follows from the fact that for any $\beta \geq 0$ we have $f\left(\lambda_{0}\right)>0$.

(2) For sufficiently large $\beta>\beta_{C}$, the solution must be a decreasing function in the region $\left(x_{1}, x_{2}\right)$. This follows from

$$
\frac{1}{2} y^{\prime}(x)=f(x)-3 \beta \sqrt{y(x)} \leq f(x)<0, \quad x_{1} \leq x \leq x_{2} .
$$

From this analysis we conclude that the solution $y(x)$ has a minimum for sufficiently large $\beta$. Denote $x_{0}$ the position of this minimum. Let us study the behavior of $y(x)$ in the neighborhood of $x_{0}$. From 


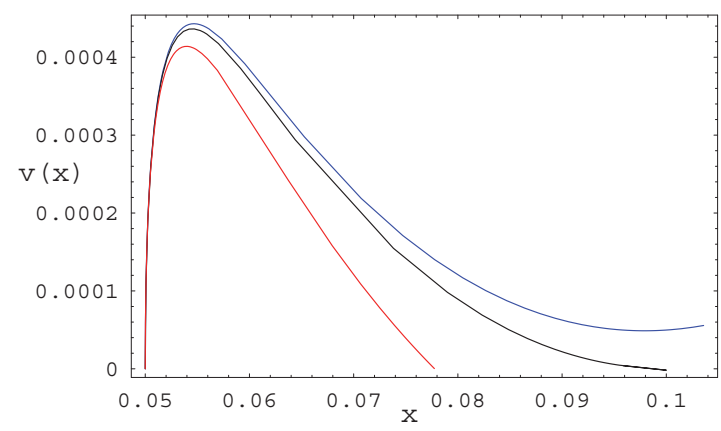

Figure 2. Numerical solutions for $v(x)$ for $\beta<\beta_{C}$ (blue), $\beta=\beta_{C}$ (black) and $\beta>\beta_{C}$ (red). The plots correspond to $\sigma=0.2, \lambda_{0}=0.05$ and $\beta=0.0625$ (blue), $\beta=\beta_{C}=0.063$ (black) and $\beta=0.066$ (red).

(35) we have

$$
3 \beta \sqrt{y\left(x_{0}\right)}=f\left(x_{0}\right) .
$$

From the properties of $f(x)$ discussed above, we see that as $\beta$ increases, $y\left(x_{0}\right)$ approaches zero as $\beta \rightarrow \beta_{\mathrm{C}}$. Thus $\lim _{\beta \rightarrow \beta_{\mathrm{C}}} x_{0}=2 \lambda_{0}$ must be the double root of $f(x)=0$ at $\beta=\beta_{C}$.

Taking one derivative of (35) with respect to $x$ we have

$$
\frac{1}{2} y^{\prime \prime}(x)+\frac{3 \beta}{2 \sqrt{y(x)}} y^{\prime}(x)=2 \sigma^{2} x-2 \beta^{2} .
$$

At $x=x_{0}$, we have $y^{\prime}\left(x_{0}\right)=0$ which gives

$$
\lim _{\beta \rightarrow \beta_{0}} \frac{1}{2} y^{\prime \prime}\left(x_{0}\right)=2 \sigma^{2} x_{0}-2 \beta^{2} .
$$

Thus we get the expansion of the solution $y(x)$ around the minimum at $x_{0}$

$$
y(x)=y\left(x_{0}\right)+2\left(\sigma^{2} x_{0}-\beta^{2}\right)\left(x-x_{0}\right)^{2}+O\left(\left(x-x_{0}\right)^{3}\right),
$$

with $y\left(x_{0}\right)=f^{2}\left(x_{0}\right) /(3 \beta)^{2}$. This shows that as $\beta \rightarrow \beta_{\mathrm{C}}$, the integral (34) diverges due to the singularity at $x=x_{0}$.

The qualitative behavior described above is seen in Figure 2 which shows numerical solutions for $v(x)$ for three values of $\beta$ around the critical value $\beta_{C}$. For $\beta<\beta_{C}$ the solution has a minimum (blue curve). For $\beta=\beta_{\mathrm{C}}$, the function $v(x)$ vanishes at $x_{0}=2 \lambda_{0}$, and its support is $x \in\left[\lambda_{0}, 2 \lambda_{0}\right]$. This is shown as the black curve in Figure 2. For $\beta>\beta_{\mathrm{C}}$ the solution vanishes at $x_{1}<2 \lambda_{0}$ (red curve).

\section{Eurodollar Futures EXPLOSION}

Consider the pricing of an Eurodollar futures contract on the rate $L(T, T+\delta)$ in the log-normal quasiGaussian HJM model. This requires the calculation of the expectation of this rate $\mathbb{E}^{\mathbb{Q}}[L(T, T+\delta)]$ in the risk-neutral measure [11]. Since the rate $L\left(T_{1}, T_{2}\right)$ is related to the zero coupon bond $P\left(T_{1}, T_{2}\right)$ as $L\left(T_{1}, T_{2}\right)=\frac{1}{T_{2}-T_{1}}\left(P^{-1}\left(T_{1}, T_{2}\right)-1\right)$, see e.g. [1], the pricing of the Eurodollar futures contract can be reduced to the evaluation of the expectation value of the inverse zero coupon bond $P^{-1}(T, T+\delta)$ in the risk-neutral measure. 
Using Eq. (4) for the zero coupon bond price $P(t, T)$ we get

$$
\mathbb{E}^{\mathbb{Q}}\left[P^{-1}(T, T+\delta)\right]=\frac{P(0, T+\delta)}{P(0, T)} \mathbb{E}^{\mathbb{Q}}\left[\exp \left(G(t, T) x_{t}+\frac{1}{2} G^{2}(t, T) y_{t}\right)\right] .
$$

This expectation is bounded from below by the Jensen inequality as

$$
\begin{aligned}
\mathbb{E}^{\mathbb{Q}}\left[\exp \left(G(t, T) x_{t}+\frac{1}{2} G^{2}(t, T) y_{t}\right)\right] & \geq \exp \left(G(t, T) \mathbb{E}^{\mathbb{Q}}\left[x_{t}\right]+\frac{1}{2} G^{2}(t, T) \mathbb{E}^{\mathbb{Q}}\left[y_{t}\right]\right) \\
& \geq \exp \left(G(t, T) \mathbb{E}^{\mathbb{Q}}\left[x_{t}\right]\right),
\end{aligned}
$$

where we recall that $x_{t}=r_{t}-\lambda(t)$. We used in the last inequality the positivity of $y_{t}$. For the smallnoise limit, and sufficiently small $\beta$, the expectation on the right-hand side explodes to infinity since we have $\lim _{t \rightarrow \tau_{\infty}} \mathbb{E}^{\mathbb{Q}}\left[r_{t}\right] \simeq \lim _{t \rightarrow \tau_{\infty}} r(t)=\infty$. We conclude thus that Eurodollar futures contract prices in this model must explode before the explosion time $\tau_{\infty}$.

The pricing of Eurodollar futures in the quasi-Gaussian HJM was considered in [4], under several volatility specifications: normal, log-normal (identical to the model considered here) and squareroot. The model was solved by finite differences methods. The numerical tests in [4] assumed a flat yield curve at $\lambda_{0}=0.05$. Several values of the rates volatility were considered $\sigma=0.05,0.1,0.3$, and mean-reversion $\beta_{1}=0.01$ and $\beta_{2}=0.1$. For all these cases one has $\beta_{C} \leq 0.1$. Typical values of the mean-reversion parameter in fixed-income markets are $\beta \leq 0.1$, so the condition $\beta<\beta_{C}$ is satisfied unless $\sigma, \lambda_{0}$ are very small, such that $\sigma \sqrt{\lambda_{0}} \leq 0.07$.

Assuming $\beta_{1} \simeq 0$, Proposition 4 gives explosion times less than or equal to $266 \mathrm{Y}, 133 \mathrm{Y}$ and $44 \mathrm{Y}$, respectively. These are much larger than typical maturities of Eurodollar futures contracts, which are listed on exchanges up to 10Y, although most of the liquid quotes are within $7 \mathrm{Y}$.

\section{Summary AND CONCLUSIONS}

We studied the qualitative behavior of the solutions of the log-normal quasi-Gaussian HJM model using a deterministic approximation, which corresponds to the small Brownian noise limit. Under this approximation, we showed that the short rate may explode to infinity in a finite time. This is relevant for the simulation and use of the model for pricing financial derivatives.

The small-noise solutions can be used to guide the construction of finite difference or tree approximations of the model SDE. The explosion phenomenon implies that Eurodollar futures prices may become infinite under certain conditions on maturity and model parameters. Explicit explosion criteria are presented, which give an upper bound on the explosion time, under weak assumptions on the shape of the initial yield curve.

\section{Appendix: A DIFFERENTIAL INEQUALity}

We prove in this Appendix that the solutions of the differential inequality (27) are bounded from below by the solution of the equation obtained by taking replacing the inequality sign with equality. Thus a sufficient condition for the explosions of the solution of (25) under Assumption 1 is that the solution with $\Lambda(t)=0$ has an explosion. On the other hand, the absence of an explosion for the solution of the latter equation does not say anything about the presence or absence of an explosion in the former case. 
Lemma 1. Denote $R(t)$ the solution of the ODE obtained by replacing the inequality sign in (27) with equality, and satisfying the initial conditions (28). The solutions of the differential inequality are bounded from below by $R(t)$

$$
r(t) \geq R(t), \quad t \geq 0
$$

Proof. Write $r(t)=R(t)+\delta(t)$ where $R(t)$ is the solution of the ODE. Taking differences gives a differential inequality for $\delta(t)$

$$
\delta^{\prime \prime}(t)+3 \beta \delta^{\prime}(t)+2 \beta^{2} \delta(t) \geq 2 \sigma^{2} R(t) \delta(t)+\sigma^{2} \delta(t)^{2} \geq 2 \sigma^{2} R(t) \delta(t) .
$$

with initial conditions $\delta(0)=0, \delta^{\prime}(0)=0$.

Define $\varepsilon(t)$ as $\delta(t)=e^{-\frac{3}{2} \beta t} \varepsilon(t)$, with initial conditions $\varepsilon(0)=0, \varepsilon^{\prime}(0)=0$. This function satisfies the inequality

$$
\varepsilon^{\prime \prime}(t) \geq F(t) \varepsilon(t)=\left(\frac{1}{4} \beta^{2} e^{\frac{3}{4} \beta t}+2 \sigma^{2} R(t)\right) \varepsilon(t),
$$

where we defined $F(t)=\frac{1}{4} \beta^{2} e^{\frac{3}{4} \beta t}+2 \sigma^{2} R(t)$.

The inequality (48) gives

$$
\varepsilon^{\prime}(t)=\int_{0}^{t} \varepsilon^{\prime \prime}(s) d s \geq \int_{0}^{t} F(s) \varepsilon(s) d s .
$$

Integrating again over $t$ we get

$$
\varepsilon(t)=\int_{0}^{t} \varepsilon^{\prime}(u) d u \geq \int_{0}^{t}\left(\int_{0}^{u} F(s) \varepsilon(s) d s\right) d u=\int_{0}^{t}(t-s) F(s) \varepsilon(s) d s,
$$

where we exchanged the order of integration in the last step. Note that $(t-s) F(s)$ is non-negative and continuous function for $0 \leq s \leq t$. Therefore by the generalized Gronwall's inequality in [9], we have $\varepsilon(t) \geq 0$.

\section{ACKNowledGements}

The authors are grateful to the editor and an anonymous referee for their helpful suggestions that greatly improved the quality of the paper. Lingjiong Zhu is grateful to the support from NSF Grant DMS-1613164. The information, views and opinions set forth in this publication are those of the authors, and are in no ways sponsored, endorsed or related to the business of J.P.Morgan Chase \& Co. ("J.P.Morgan"). J.P.Morgan does not warrant the publication's completeness or accuracy, and makes no representations regarding the use of the information set forth in this publication. In no event shall J.P.Morgan be liable for any direct, indirect, special, punitive, or consequential damages, including loss of principal and/or lost profits, even if notified of the possibility of such damages. Nothing in this publication is intended to be an advertisement or offer for any J.P.Morgan service.

\section{REFERENCES}

[1] Andersen, L. and V. Piterbarg. Interest Rate Modeling. Atlantic Press, London, 2010.

[2] Andreasen, J. Markovian term structure models, in Encyclopedia of Quantitative Finance, Ed. R. Cont, Wiley, New York, 2010.

[3] Babbs, S. (1993). Generalized Vasicek models of the term structure, Applied Stochastic Models and Data Analysis. 1, 49-62. 
[4] Cakici, N. and J. Zhu. (2001). Pricing Eurodollar futures options with the Heath-Jarrow-Morton model. Journal of Futures Markets. 21, 655-680.

[5] Cheyette, O. (1992). Markov representation of the Heath-Jarrow-Morton model. Working paper, Barra.

[6] Chibane, M. (2012) Explicit volatility specification for the linear Cheyette model. Working paper, Shinsei Bank Ltd.

[7] Chibane, M. and D. Law (2013). A quadratic volatility Cheyette model. Risk, 60-63 (July).

[8] Chien, H. H.(2001). On the complexity of the Ritchken Sankarasubramanian interest rate model. Ph.D. Thesis, National Taiwan University.

[9] Chu, S. and F. Metcalf. (1967). On Gronwall's Inequality. Proc. Amer. Math. Soc. 18, 439-440.

[10] Heath, D., R. Jarrow and A. Morton. (1992). Bond pricing and the term structure of interest rates: A new methodology for contingent claim valuation. Econometrica. 60, 77-105.

[11] Hunt, P. J. and J. E. Kennedy. Financial Derivatives in Theory and Practice. Wiley Series in Probability and Statistics, 2004.

[12] Jamshidian, F. (1991). Bond and option evaluation in the Gaussian interest rate model. Research in Finance. 8, 131-170.

[13] Morton, A. J. (1989). Arbitrage and martingales. Doctoral dissertation, Cornell University.

[14] Pirjol, D. and L. Zhu. (2016). Explosion of the quasi-Gaussian log-normal HJM model. Working paper.

[15] Ritchken, P. and L. Sankarasubramanian. (1995). Volatility structures of forward rates and the dynamics of the term structure. Math. Finance. 5, 55-72.

[16] Whittaker, E. T. and G. N. Watson (1927). A Course of Modern Analysis. Cambridge University Press.

J. P. MORGAN

277 Park Avenue

NEW YORK, NY-10172

United States of America

E-mail address: dpirjol@gmail.com

Department of Mathematics

Florida State University

1017 ACADEMIC WAY

Tallahassee, FL-32306

United States of America

E-mail address: ling@cims.nyu.edu 
Figure

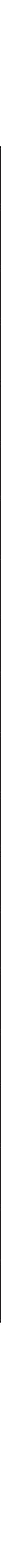




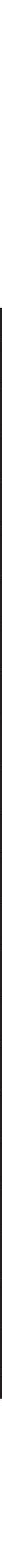

Figure

Figure

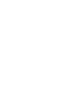

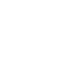

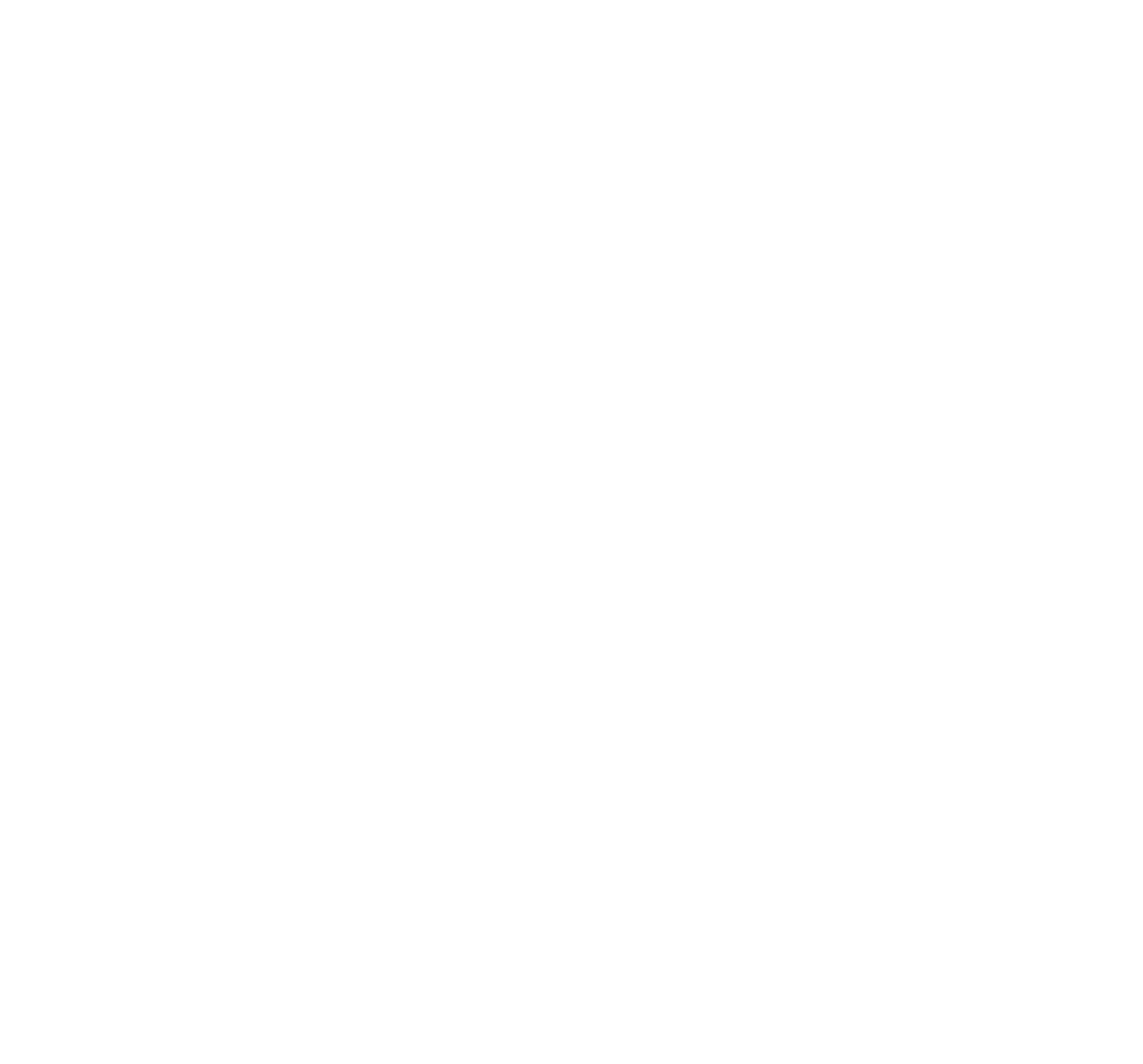

